\title{
Rigidity Theorems in Riemannian geometry
}

\author{
Christopher B. Croke*
}

May 30, 2002

\section{Introduction}

The purpose of this chapter is to survey some recent results and state open questions concerning the rigidity of Riemannian manifolds. The starting point will be the boundary rigidity and conjugacy rigidity problems. These problems are connected to many other problems (Mostow-Margulis type rigidity, isopectral problems, isoperimetric inequalities etc.). We will restrict our attention to those results that have a direct connection to the boundary rigidity problem (see section 2) or the conjugacy rigidity problem (see section 4 ). Even with that restriction the connections are numerous and the author was forced to select the topics covered here in accordance with rather subjective criteria. A few of the topics not covered are mentioned in section 11 but there are others.

Although this chapter consists mostly of a survey of known results there are a few results that appear here in print for the first time. In particular, many of the applications of the Besson-Courtois-Gallot minimal entropy theorem that occur in section 6.1 are new. Also the applications of the Pestov-Sharafutdinov theorem in section 8 (which are due to Kleiner and the author) appear here for the first time. Most of the proofs are omitted and the reader is referred to the original articles. The exceptions are when the results are in print for the first time or when they are short and instructive.

The author would like to thank Bruce Kleiner for feedback on the first draft of this chapter. He would also like to thank Chang-Wan Kim for a careful reading of an earlier draft.

\section{Contents}

1 Introduction . . . . . . . . . . . . . . . . . 1

2 The Boundary rigidity problem . . . . . . . . . . . . 2

3 Inverse kinematic problem of seismology . . . . . . . . . . . . 5

4 Conjugacy rigidity and related problems . . . . . . . . . . . 7

5 Nonpositive curvature in two dimensions . . . . . . . . . . . . . . . 9

*Supported by NSF grant DMS 99-71749 and CRDF grant ????. 
6 Symmetric Spaces of negative curvature . . . . . . . . . . . . . . . 10

7 Nonpositive curvature in higher dimensions . . . . . . . . . . . . . . . 14

8 The linearized and local problem . . . . . . . . . . . . . 16

9 Products . . . . . . . . . . . . . . . . . . . 20

10 No conjugate points . . . . . . . . . . . . . . . . . 21

11 Some Other Related Topics . . . . . . . . . . . . . . . . . 22

\section{The Boundary rigidity problem}

Let $(M, \partial M, g)$ be a compact connected Riemannian manifold with smooth boundary. Then $\partial M$ inherits a "boundary distance function" $d_{g}$, i.e., $d_{g}(p, q)$ is the "chordal" distance in $M$ between boundary points $p$ and $q$. Since $d_{g}$ is (typically) sensitive to the geometry of the interior of $M$, one may ask to what extent the metric space $(M, \partial M, g)$ determines $M$ up to isometry; this is the Boundary rigidity problem. We formalize the problem as follows. If $(M, \partial M, g)$ and $\left(M_{1}, \partial M_{1}, g_{1}\right)$ are Riemannian manifolds with boundary distance function $d_{g}$ and $d_{g_{1}}$ respectively, we call an isometry $(\partial M, d g) \rightarrow$ $\left(\partial M_{1}, d_{g_{1}}\right)$ a boundary equivalence. A compact connected manifold $(M, \partial M, g)$ is boundary rigid if whenever $\left(M_{1}, \partial M_{1}, g_{1}\right)$ is compact connected then any boundary equivalence $(M, \partial M, g) \rightarrow\left(M_{1}, \partial M_{1}, g_{1}\right)$ extends to an isometry $(M, g) \rightarrow\left(M_{1}, g_{1}\right)$.

All manifolds in this chapter are assumed to be connected, however the boundaries of manifolds with boundary are not always connected. When $(\partial M, d g)$ and $\left(\partial M_{1}, d_{g_{1}}\right)$ are boundary equivalent we can (and will) identify $\partial M_{1}$ with $\partial M$ and say $d_{g}=d_{g_{1}}$.

One cannot expect that all compact manifolds with boundary will be boundary rigid. Examples of non boundary rigid manifolds can be easily constructed by choosing a metric so that no minimizing geodesic from boundary point to boundary point passes through a given point $x$ (e.g. make the distance from $x$ to $\partial M$ greater than the diameter of $\partial M$ as a subset of $M$ ). In this case a change of metric (or even topology) in a small neighborhood of $x$ will yield a metric $g_{1}$ not isometric to $g$ but with $d_{g}=d_{g_{1}}$. The round hemisphere also provides an example since any metric which is larger than the standard metric and agrees on the boundary will have the same boundary distance function. More interesting counter examples are provided by surfaces of revolution and their higher dimensional generalizations (see [We] and [Cr-Kl1] which we will discuss below in section 4). But there are some boundary rigid manifolds. For constant curvature spaces we have:

Theorem 2.1. Let $\left(M^{n}, \partial M, g\right)$ be a compact subdomain with smooth boundary $\partial M$ of Euclidean space $\mathbb{R}^{n}$, hyperbolic space $H^{n}$, or the open hemisphere of $S^{n}$. Then $(M, \partial M, g)$ is boundary rigid.

The hemisphere case was proved by Michel [Mi] (also see section 4) using the solution to the Baschke conjecture for spheres (see [Be-Ka]), the Euclidean space case was proved by Gromov (see section 5.5B of [Gro] or alternatively [Cr2]). The hyperbolic space case can be proved from the minimal entropy theorem of [B-C-G2] (see section 6 for a discussion). In fact, the results extend somewhat beyond the subdomain case (e.g. in the flat case one needs only assume that there is an isometric immersion of 
$\left(M^{n}, \partial M, g\right)$ to $\mathbb{R}^{n}$ - the reader should consult the original sources for such extensions). The only spaces of nonconstant curvature that are known to be boundary rigid are two dimensional spaces of nonpositive curvature (see section 5), subdomains of negatively curved symmetric spaces (see section 6), and some products (see section 9).

Because of the counterexamples we need to restrict the class of manifolds with boundary that we consider. It is important that one can determine whether $(M, \partial M, g)$ belongs to the class by its boundary distance function $d$ since we will then know that any $\left(M_{1}, \partial M_{1}, g_{1}\right)$ which is boundary equivalent to $(M, \partial M, g)$ will also be in that class.

There are two natural (for different reasons) such classes of $(M, \partial M, g)$ we consider, the "simple" case and the "SGM" (strong geodesic minimizing) case.

$(M, \partial M, g)$ will be called simple if the boundary is strictly convex and there is a unique geodesic between any pair of boundary points. It is not hard to see (by looking at geodesics leaving a fixed boundary point) that topologically $M$ is a ball and that this condition is determined by the boundary distance function. The simplicity condition will guarantee that certain problems are elliptic, and also has the advantage that any other metric $g_{1}$ with $d_{g}=d_{g_{1}}$ will have the same $C^{\infty}$ jet as $g$ at $\partial M$. (This was proved by Michel for the $C^{2}$ jet. For the $C^{\infty}$ jet see [L-S-U].)

The SGM condition (which is given in terms of $d_{g}$ only) is harder to define. A precise definition is given in [Cr1], but loosely speaking it means that all nongrazing geodesic segments are strongly minimizing. By a nongrazing geodesic segment we will mean a segment of a geodesic which lies in the interior of M except possibly for the endpoints. A segment is said to minimize if its length is the distance between the endpoints and to strongly minimize if it is the unique such path. Of course this loose definition seems to rely on more than $d_{g}$ but the relationship is worked out in [Cr2]. Examples of such $(M, \partial M, g)$ are given by compact subdomains of an open ball, $\mathrm{B}$, in a Riemannian manifold where all geodesics segments in B minimize - in particular all the spaces in Theorem 2.1.

Before going on much longer I should pose the question that is at the heart of the matter:

Question 2.2. Let $g_{0}$ be a Riemannian metric on $B$, the unit ball in $\mathbb{R}^{n}$, such that all geodesics minimize distance, and the distance from the origin to any point on the boundary sphere is 1 . (You might further assume that the boundary is strictly convex or that the curvature is negative.) Then if $g_{1}$ is any other Riemannian metric on $B$ with $d_{g_{1}}=d_{g_{0}}$ must there be a point $p \in B$ such that the minimum $g_{1}$-distance from $p$ to the boundary is 1 .

The only cases where this question has been answered are cases where $g_{0}$ is shown to be isometric to $g_{1}$ and $p$ is then the point corresponding to the origin. A positive answer to this question for a large class of $g_{0}$ would yield boundary rigidity in a correspondingly large class. The proof of this conclusion is not too hard but we will omit it here.

For a Riemannian manifold with boundary $(M, \partial M, g)$ we will let $U^{+} \partial M$ represent the open disc bundle of unit vectors at boundary points that point inwards into $M$. 
Note that orthogonal projection allows us to identify these vectors with the open unit disc bundle of $\partial M$ (i.e. the vectors tangent to $\partial M$ whose length is less than one). Similarly define as $U^{-} \partial M$ those that point out. Thus if $\left(M_{1}, \partial M, g_{1}\right)$ is a Riemannian metric so that $\left.g\right|_{\partial M}=\left.g_{1}\right|_{\partial M}$ (in particular if $d_{g}=d_{g_{1}}$ ) then we can identify the $U^{+} \partial M$ (and the $U^{-} \partial M$ ) from the two metrics.

We will say that $(M, \partial M, g)$ is lens equivalent to $\left(M_{1}, \partial M, g_{1}\right)$ if for each $V \in$ $U^{+} \partial M$ the entering $g$-geodesic, $c_{V}(t)$ with $c_{V}^{\prime}(0)=V$ and the entering $g_{1}$-geodesic $c_{1 V}(t)$ with $c_{1 V}^{\prime}(0)=V$ exit in the same way in the sense that if $t_{0}$ is the first time, $t>0$ such that $c_{V}(t) \in \partial M$ then the same is true for $c_{1 V}$, and further $c_{V}\left(t_{0}\right)=c_{1 V}\left(t_{0}\right)$ and $c_{V}^{\prime}\left(t_{0}\right)=c_{1 V}^{\prime}\left(t_{0}\right)$. That is if we think of the two spaces acting as lenses with geodesics as light rays, then they bend the light in exactly the same way.

It is easy to see that if $(M, \partial M, g)$ is lens equivalent to $\left(M_{1}, \partial M, g_{1}\right)$ then $d_{g}=d_{g_{1}}$. It is also not hard to see that if $(M, \partial M, g)$ is simple or SGM (and hence so is $\left.\left(M_{1}, \partial M, g_{1}\right)\right)$ and $d_{g}=d_{g_{1}}$ then $(M, \partial M, g)$ is lens equivalent to $\left(M_{1}, \partial M, g_{1}\right)$. The easiest way to see this is to first consider the metric $g$ and a fixed $p \in \partial M$ and consider the functions $f_{p}: M \rightarrow \mathbb{R}$ defined by $f_{p}(x)=d(p, x)$ and $h_{p}: \partial M \rightarrow \mathbb{R}$ by $h_{p}(q)=d(p, q)$. Now consider $q_{0} \in \partial M$ such that the minimizing geodesic from $p$ to $q_{0}$ does not graze. We see that $\nabla f_{p}\left(q_{0}\right)$ is the (outward or tangent) unit vector tangent to the geodesic from $p$ to $q_{0}$. But since $\left.f_{p}\right|_{\partial M}=h_{p}$ we see that the orthogonal projection of $\nabla f_{p}\left(q_{0}\right)$ is just $\nabla h_{p}\left(q_{0}\right)$. Now since $h_{p}$ (and hence $\nabla h_{p}\left(q_{0}\right)$ ) is the same for the metric $g_{1}$ we see that the exiting for the $g_{1}$-geodesic from $p$ to $q_{0}$ agrees with that of $g$. Reversing the roles of $p$ and $q_{0}$ we see that the geodesics have the same initial tangents as well. This will allow us to conclude that the metrics are lens equivalent. Thus the boundary rigidity question is closely related to the corresponding lens rigidity question.

Given $(M, \partial M, g)$ and $\left(M_{1}, \partial M, g_{1}\right)$ such that $\left.g\right|_{\partial M}=\left.g_{1}\right|_{\partial M}$ then a geodesic conjugacy is a is a $C^{k}$ diffeomorphism $F: U M \rightarrow U M_{1}$ between the unit tangent bundles such that unit vectors based at boundary points go to corresponding unit vectors (as discussed above), and such that $g_{M_{1}}^{t} \circ F=F \circ g_{M}^{t}$ wherever either (and hence both) sides are defined. In the above $g^{t}$ represents the geodesic flow, i.e. for a unit vector, $V, g^{t}(V)=c_{V}^{\prime}(t)$ where $c_{V}$ is the geodesic with $c_{V}^{\prime}(0)=V$. In both the simple and the SGM cases every geodesic in the interior of $M$ is a subset of a minimizing geodesic segment between two boundary points. Thus if $d_{g}=d_{g_{1}}$ then we can define (uniquely) such a geodesic conjugacy as follows. For $V \in U M$ let $-t_{0}$ be the smallest $t \leq 0$ such that $c_{V}(t) \in \partial M$ and let $W=c_{V}^{\prime}\left(-t_{0}\right)$ and hence $g_{M}^{t_{0}}(W)=V$. We thus, using again our identification of unit vectors of the two metrics at boundary points, define $F(V)=g_{M_{1}}^{t_{0}}(W)$.

The fact that $F$ is well-defined follows from lens equivalence. In the simple case $F$ will be $C^{\infty}$, while in the SGM case $F$ will be $C^{0}$ and $C^{\infty}$ off a set of measure 0 . It follows directly from the definition that $F(-V)=-F(V)$, however one cannot assume that $F$ descends to a map $f: M \rightarrow M_{1}$ (i.e., $F$ may take two vectors based at the same interior point to vectors with different base points). In fact if there is such an $f$ where $F$ takes each unit vector based at $x \in M$ to a unit vector at $f(x)$ then $f$ would have to be an isometry and $F=D f$. To see this simply note that, by looking at the $F$ image of the tangent vector field of geodesic segments, $f$ would have to take each geodesic segment to a geodesic segment of the same length. 
It is also not hard to see that such a geodesic conjugacy will preserve the canonical contact form and hence the Liouville volume form. And thus we get:

Lemma 2.3. If $(M, \partial M, g)$ is simple or $S G M$ and $\left(M_{1}, \partial M_{1}, g_{1}\right)$ is boundary equivalent to $(M, \partial M, g)$ then $\operatorname{Vol}(g)=\operatorname{Vol}\left(g_{1}\right)$.

One can also see the above result from Santaló's formula (see section 3).

Since having conjugate geodesic flows is a stronger condition in general than having the same boundary distance function one might hope to get rigidity for a larger class of metrics than for the simple or SGM cases. However, there are counterexamples based on the Zoll surfaces of revolution [We]. Specifically, examples were created in [Cr-Kl1] of nonisometric metrics on the $n$-ball for which there is a geodesic conjugacy as above (in particular the metrics and conjugacy agree on the boundary) that preserves the standard contact forms.

In what follows there are many results where some extra assumption (usually a curvature condition) is placed on the manifold $\left(M_{1}, \partial M_{1}, g_{1}\right)$. The expression $(M, \partial M, g)$ is boundary rigid among negatively curved spaces will mean that for any $\left(M_{1}, \partial M_{1}, g_{1}\right)$ of negative curvature then any boundary equivalence $(M, \partial M, g) \rightarrow\left(M_{1}, \partial M_{1}, g_{1}\right)$ extends to an isometry $(M, g) \rightarrow\left(M_{1}, g_{1}\right)$. We will use similar expressions for other curvature type conditions.

\section{Inverse kinematic problem of seismology}

The history of the boundary rigidity problem goes back to 1905 to papers by Herglotz [Her], and Wiechert and Zoepprtz [Wi-Zo] where they consider the case of radially symmetric metrics on the ball $B^{3}$ as a model of the earth. The next advances were due to Romanov [Ro1] who considered the linearized problem about radial metrics. In particular, he found in the radially symmetric case that you could determine a function from its integrals along geodesics.

The next advance concerned isotropic metrics, i.e., $g_{1}=\lambda(x) g_{0}$, where $\lambda$ is a smooth positive function on the manifold. In the case of simple metrics the major advances were made by Mukhometov. He proved the two dimensional case in 1975 [Mu1, Mu2] (also see [Ar-Mi]) and the higher dimensional case in 1982 [Mu3]. His results also contain nice stability estimates. Others independently proved such results (for example see [Ro2]).

The case when $M$ is SGM was resolved in [Cr2]. The proof is easy enough so we present it here along with a stability estimate. There is a canonical measure $d \gamma$ on the space of geodesic segments (which in our case run from a boundary point to a boundary point) $\Gamma_{0}$ such that for every integrable function $f$ on the unit tangent bundle we have:

$$
\int_{\Gamma_{0}} \int_{0}^{L(\gamma)} f\left(\gamma^{\prime}(t)\right) d t d \gamma=\int_{U M} f(u) d u .
$$

In our case we can parameterize the geodesic segments by their initial tangents $v \in$ $U^{+} \partial M$ where $U^{+} \partial M$ represents the unit tangent vectors to $M$ whose base points are on $\partial M$ and which point "inward" (i.e., $\langle v, N>\geq 0$ where $N$ is the inward normal). 
Santaló's formula (which is used in the proof of many of the results we are discussing in this paper) allows us to express $d \gamma$ as $\langle N, v\rangle d v$ where $d v$ is the measure which locally is the product of the Riemannian measure on the base point with the standard measure on a unit hemisphere. (All of the above have versions in the non SGM case.)

We let $M$ be an $n$-dimensional manifold with boundary such that there two $S G M$ metrics: $g_{0}$ with volume $V_{0}=\int_{M} d x_{0}$ and $g_{1}=\lambda g_{0}$ of volume $V_{1}=\int_{M} \lambda^{n} d x_{0}$. We let $\Gamma_{0}$ be the space of unit speed geodesic segments in $M_{0}$ (with endpoints in $\partial M)$ with the usual measure. For $\gamma \in \Gamma_{0}$ and $i=1,2$, we let $L_{i}(\gamma)$ represent the length of $\gamma$ in the $g_{i}$ metric while $\mathcal{L}_{i}(\gamma)$ will represent the distance in $g_{i}$ between the endpoints of $\gamma$. Of course $L_{0}(\gamma)=\mathcal{L}_{0}(\gamma)$ while $L_{1}(\gamma) \geq \mathcal{L}_{1}(\gamma)$. Note that $L_{1}(\gamma)=\int_{0}^{L_{0}(\gamma)} \lambda(\gamma(t)) d t$. Further, by applying equation 3.1 with $f \equiv 1$ we have $\alpha(n-1)^{-1} \int_{\Gamma_{0}} L_{0}(\gamma) d \gamma=\int_{M} d x=V_{0}$ where $\alpha(n-1)$ is the volume of the unit $n-1$ sphere. (This gives our alternative proof of Lemma 2.3). In what follows $c_{1}=\alpha(n-1)^{-1}$.

By a Hoelder inequality:

$$
\int_{M} \lambda^{2} d x_{0} \leq\left\{\int_{M} \lambda^{n} d x_{0}\right\}^{\frac{2}{n}}\left\{\int_{M} d x_{0}\right\}^{\frac{n-2}{n}}=V_{1}^{\frac{2}{n}} V_{0}^{\frac{n-2}{n}} .
$$

Using equation 3.1 (twice) we see:

$$
\begin{aligned}
\int_{M} \lambda d x_{0} & =c_{1} \int_{\gamma \in \Gamma_{0}} \int \lambda(\gamma(t)) d t d \gamma_{0}=c_{1} \int_{\gamma \in \Gamma_{0}} L_{1}(\gamma) d \gamma_{0} \\
& =c_{1} \int_{\gamma \in \Gamma_{0}} L_{0}(\gamma) d \gamma_{0}+c_{1} \int_{\gamma \in \Gamma_{0}}\left(L_{1}(\gamma)-L_{0}(\gamma)\right) d \gamma_{0} \\
& =\int_{M} d x_{0}+c_{1} \int_{\gamma \in \Gamma_{0}}\left(L_{1}(\gamma)-L_{0}(\gamma)\right) d \gamma_{0}
\end{aligned}
$$

and hence

$$
2 \int_{M}(1-\lambda) d x_{0}=2 c_{1} \int_{\gamma \in \Gamma_{0}}\left(L_{0}(\gamma)-L_{1}(\gamma)\right) d \gamma_{0} \leq 2 c_{1} \int_{\gamma \in \Gamma_{0}} \mathcal{L}_{0}(\gamma)-\mathcal{L}_{1}(\gamma) d \gamma_{0}
$$

Thus since

$$
\int_{M}(1-\lambda)^{2} d x_{0}=\int_{M} \lambda^{2} d x_{0}+2 \int_{M}(1-\lambda) d x_{0}-V_{0}
$$

using 3.2 and 3.3 we arrive at the stability estimate:

$$
\int_{M}(1-\lambda)^{2} d x_{0} \leq\left(V_{1}^{\frac{2}{n}}-V_{0}^{\frac{2}{n}}\right) V_{0}^{\frac{n-2}{n}}+2 c_{1} \int_{\gamma \in \Gamma_{0}} \mathcal{L}_{0}(\gamma)-\mathcal{L}_{1}(\gamma) d \gamma_{0}
$$

In particular if $\mathcal{L}_{0}=\mathcal{L}_{1}$ then also $V_{0}=V_{1}$ and we get the boundary rigidity result that $\lambda \equiv 1$. Another consequence is that if $\mathcal{L}_{1} \geq \mathcal{L}_{0}$ (i.e $d_{g_{1}} \geq d_{g_{0}}$ ) then $V_{1} \geq V_{0}$. The difficulty in using this stability estimate is that although $V_{i}$ is determined by $\mathcal{L}_{i}$ via $V_{i}=c_{1} \int_{U_{i}^{+} \partial M} L_{i}\left(\gamma_{v}\right)<N, v>d v$ (where $\gamma_{v}$ here is the geodesic in $g_{i}$ ), in order to determine how $v$ depends on the endpoints involves the derivatives of $\mathcal{L}_{i}$. 


\section{Conjugacy rigidity and related problems}

One way of finding manifolds that are boundary rigid is to look at subdomains (simple or SGM) of closed (or more generally complete) Riemannian manifolds without boundary that are geometrically rigid in some way and using a cutting and pasting argument. In particular, let $\left(M, \partial M, g_{0}\right)$ is a compact subset of a complete Riemannian manifold $\left(N, g_{0}\right)$ without boundary. If $\left(M_{1}, \partial M, g_{1}\right)$ (here $M_{1}$ need not a-prioiri be diffeomorphic to $M$ but must have the same boundary $\partial M$ ) has $d_{g}=d_{g_{1}}$ then we can create a new complete Riemannian manifold $\left(N_{1}, g_{1}\right)$ by cutting $M$ out of $N$ and replacing it by $N_{1}$. If $\left(M, \partial M, g_{0}\right)$ is simple then the new metric $g_{1}$ on $N_{1}$ will in fact be smooth (as was pointed out in section 2). However, in the SGM case we only get $C^{0}$ and $C^{\infty}$ on $N-\partial M$, but this is often enough differentiability for our purposes. Now if $\left(N, g_{0}\right)$ has the right type of rigidity we may be able to conclude that $g_{0}$ is isometric to $g_{1}$ and hence that $\left(M, \partial M, g_{0}\right)$ is boundary rigid. In fact most cases where boundary rigidity has been proved have been proved this way.

For example (Michel $[\mathrm{Mi}])$, let $\left(M, \partial M, g_{0}\right)$ be a subdomain of the standard $n$ sphere such that $M \cap-M=\emptyset$ (here $-M$ is the image of $M$ under the antipodal map). If $\left(M_{1}, \partial M, g_{1}\right)$ has $d_{g}=d_{g_{1}}$ then we can do the cut and paste to both $M$ and $-M$ to get a new Riemannian manifold such that for every point $p$ all geodesics leaving $p$ minimize to length $\pi$, and pass through a point $-p$ at length $\pi$. This is a so called Blaschke metric and is isometric to the standard sphere since the Blaschke conjecture for spheres has been solved $([\mathrm{Be}-\mathrm{Ka}])$ and the proof is robust enough to work for metrics with the type of differentiability we get. Thus such $\left(M, \partial M, g_{0}\right)$ are boundary rigid. The same type of argument might be tried for subdomains of the other rank one symmetric spaces of positive curvature, but the corresponding Blaschke conjectures are still open.

Definition 4.1. If $M$ and $M^{\prime}$ are Riemannian manifolds without boundary a map $F: U M^{\prime} \rightarrow U M$ between the unit tangent bundles is called a $C^{k}$ conjugacy between the geodesic flows if it is a $C^{k}$ diffeomorphism and $g_{t}^{M} \circ F=F \circ g_{t}^{M^{\prime}}$ where $g_{t}^{M^{\prime}}$ (resp. $\left.g_{t}^{M}\right)$ is the geodesic flow on $M^{\prime}$ (resp. $\left.M\right) . \mathcal{F}^{k} U(M)$ will refer to the $C^{k}$ conjugacy class of the geodesic flow on $M$. That is, $M^{\prime} \in \mathcal{F}^{k} U(M)$ if there is a $C^{k}$ conjugacy between the geodesic flows of $M$ and $M^{\prime}$. $M$ is called $C^{k}$ conjugacy rigid if it is isometric to all spaces $M^{\prime} \in \mathcal{F}^{k} U(M)$.

The cutting and pasting argument above allows us to conclude:

Lemma 4.2. Simple subdomains of a $C^{\infty}$ conjugacy rigid manifold are boundary rigid.

Examples of $C^{\infty}$ conjugacy rigid manifolds are given by $R P^{n}$ with the standard metric (by the Blaschke conjecture for spheres [Be-Ka]), compact surfaces of nonpositive curvature (see section 7), compact flat manifolds, compact locally symmetric spaces of negative curvature and some compact locally symmetric metrics built out 
of such products (see section 6), and compact manifolds that admit a parallel vector field (see section 9). This gives new classes of manifolds with boundary that are boundary rigid.

Not all compact manifolds without boundary are $C^{\infty}$ boundary rigid. In fact for any given smooth manifold $M$ one can put one of the metrics from [Cr-Kl1] on a smooth ball $B$ in $M$ and extend it arbitrarily to the rest of $M$. One can now deform the metric nontrivially on this ball keeping $C^{\infty}$ conjugacy. That is, there is a 1 parameter family of metrics $g_{t}$ such that $g_{0}$ is the original metric, $g_{t}$ agrees with $g_{0}$ outside $B$, the geodesic flow of $g_{t}$ is $C^{\infty}$ conjugate to the geodesic flow of $g_{0}$ (preserving the contact form), but $g_{t}$ is not isometric to $g_{0}$.

Some properties are always preserved under $C^{k}$ conjugacies. $C^{1}$-conjugacies always preserve the volume [Cr-Kl1]. It is still not known if $C^{0}$ conjugacies do (even in the negative curvature setting - but see [Ha2] when one metric is locally symmetric). It is also known that the property "no conjugate points" (see Section 10) is preserved under $C^{0}$ conjugacies [Cr-Kl2]

We now give the definitions of some invariants of a compact Riemannian manifold $M$ without boundary and some of their relations. We will be concerned with the extent to which the metric on $M$ is determined by these invariants.

\section{Definition 4.3.}

The Spectrum, $\operatorname{Spec}(M)$, is the eigenvalue spectrum of the Laplace Beltrami operator (counted with multiplicity).

The Length Spectrum, Lspec $(M)$, is the set of all lengths of closed geodesics of $M$ (multiplicity is not counted). When $M$ has nonpositive curvature (or more generally no conjugate points) then all the closed geodesics in a given homotopy class have the same length.

The Marked length Spectrum, MLspec $(M)$, refers to the map which assigns to each conjugacy class in $\pi_{1}(M)$ the length of the shortest closed geodesic in that free homotopy class. Hence, in the case of nonpositive curvature, $\operatorname{Lspec}(M)$ is just the image of $\operatorname{MLspec}(M)$.

These invariants are closely related to each other. In the case where $M$ has negative curvature for example we know by $[\mathrm{Du}-\mathrm{Gu}]$ that $\operatorname{Spec}(M) \operatorname{determines} \operatorname{Lspec}(M)$ (the number of closed geodesics of a given length is not necessarily determined), further $\mathcal{F}^{0} U(M)$ determines and is determined by $\operatorname{MLspec}(M)$ (see [Ha1]), and of course $\operatorname{MLspec}(M)$ determines $\operatorname{Lspec}(M)$. Whether, in the negative curvature case, $\operatorname{MLspec}(M)$ determines $\mathcal{F}^{1} U(M)$ is an open (and important) question. The 2-dimensional case was solved in $[\mathrm{Fe}-\mathrm{Or}]$.

The volume of $M$ is determined by $\operatorname{Spec}(M)$, but it is not known (and it is an important question) if the volume is determined by $\operatorname{Lspec}(M), \operatorname{MLspec}(M)$, or $\mathcal{F}^{0} U(M)$. On the other hand, as we mentioned above, the volume is always determined by $\mathcal{F}^{1} U(M)$.

There are by now a number of examples of isospectral, nonisometric manifolds (including 2-dimensional constant negative curvature ones by Vignéras [Vi]). The 
negatively curved examples thus also have the same length spectrum. On the other hand (see section 5) surfaces (2-dimensional), $M$ and $M^{\prime}$, of negative curvature with $\operatorname{MLspec}(M)=\operatorname{MLspec}\left(M_{1}\right)$ must be isometric.

A major open question in this area is the generalization to higher dimensions:

Question 4.4. Are compact negatively curved manifolds determined by their geodesic flows or their marked length spectra?

In negative curvature all of the above rigidity questions "linearize" to the same statement. This becomes: If $g_{0}$ is a metric of negative curvature on a compact manifold and $g_{t}$ a smooth 1-parameter family of metrics with the same length spectrum (hence the same marked length spectrum by continuity) then $g_{t}$ is isometric to $g_{0}$. This question was resolved in [Cr-Sh] (see section 8). In contrast, without the curvature assumption there are by now many examples of nontrivial isospectral deformations. The first of these was given in [Go-Wi] and the chapter by Ballmann in this book will describe some recent developments. Also see section 11.

Although there are thus no nontrivial 1-parameter families of negatively curved manifolds:

Question 4.5. Can there be infinitely many nonisometric compact isospectral manifolds of (pinched?) negative curvature?

One would approach the question by studying the space of isospectral metrics (in the $C^{k}$ topology) in two parts; compactness, and "local" uniqueness. Some work has been done on the compactness part (for example [Ch-Ya, B-P-P, An, Br]). By "local" uniqueness we mean uniqueness among metrics in some $C^{k}$ neighborhood of our given metric. Of course, the inherent infinite dimensionality of the problem makes the local uniqueness problem quite a bit harder than the linearized (i.e., infinitesimal

uniqueness) problem. See section 8 and the chapter by Sharafutdinov for some results of this type.

\section{Nonpositive curvature in two dimensions}

Other than the conformal case discussed in section 3 the other case where a large class of manifolds are known to be boundary rigid or conjugacy rigid is in two dimensions when the curvature is non-posiitve. The initial work was done independently by Otal (see [Ot1] and [Ot2]) and the author (see [Cr3]) under a negative curvature assumption and was extended to the nonpositive case (using the method of Otal) in $[\mathrm{C}-\mathrm{F}-\mathrm{F}]$. The best statements in the literature are:

Theorem 5.1. Every compact two dimensional Riemannian manifold (without boundary) of nonpositive curvature is $C^{0}$ conjugacy rigid. 
And in fact:

Theorem 5.2. If $g$ and $g_{1}$ are Riemannian metrics on a compact two dimensional manifold (without boundary) having the same marked length spectrum where $g$ has nonpositive curvature and $g_{1}$ has no conjugate points then $g$ is isometric to $g_{1}$.

One gets as a corollary that every SGM subdomain of such a surface is boundary rigid. Further:

Theorem 5.3. Every SGM two dimensional Riemannian manifold of negative curvature is boundary rigid.

Underlying all these results is the Gauss Bonnet Theorem which seems to be why the arguments have not extended to higher dimensions. One of the fundamental observations in Otal's method is to notice that if three geodesics pass through a point in $M^{\prime}$ then the sum of the angles between them is $\pi$ while if the corresponding geodesics (say via the conjugacy) in the negatively curved space $M$ do not pass through a point then they will form a geodesic triangle where the sum of the angles is $\leq \pi$ by the Gauss-Bonnet Theorem and will be strictly less then $\pi$ unless the triangle has no area (or in the nonpositive curvature case has integral of curvature $=0$ ). By integrating this information appropriately one forces all such triangles to be degenerate forcing the conjugacy to cover a map on the base which must be an isometry by the discussion in section 2 .

The argument in [Cr3] involved the study of Jacobi fields. Jacobi fields are vector fields along a geodesic that arise as the variation fields to variations through geodesics. Since a $C^{1}$ conjugacy will give a correspondence between variations of geodesics it also gives a correspondence between Jacobi fields. Besides the Gauss-Bonnet Theorem, [Cr3] used the fact that if $J(t)$ is a Jacobi field along a geodesic of $M$ such that $J(0)=0$ then the corresponding Jacobi field $\bar{J}$ satisfies $\bar{J}\left(t_{0}\right)=0$ for some $t_{0}$ (it is just the intermediate value theorem). In higher dimensions this no longer clear (though some such Jacobi fields must vanish). However, when $n \geq 3$ if all such $\bar{J}$ vanish somewhere than one can show that $M$ and $M^{\prime}$ are isometric. So in some sense this vanishing is the crux of the mater.

Cao in [Cao] took up the noncompact finite volume case. He showed that if two orientable, uniform visibility surfaces of finite area and bounded non-positive curvature have the same marked length spectrum, then they must be isometric.

\section{Symmetric Spaces of negative curvature}

In this section we introduce some new manifolds with boundary that are boundary rigid. The main class are the subdomains of symmetric spaces of negative curvature. The fact that these spaces are boundary rigid follow more or less directly from the equality case in the minimal entropy theorem of Besson-Courtois-Gallot (see [B-C-G1] or [B-C-G2]) which we now explain. 
A symmetric space $M$ is a Riemannian manifold such that for every point $p \in M$ the geodesic symmetry $I_{p}: M \rightarrow M$ at $p$ is an isometry. $I_{p}$ is the map (in general only locally defined - but defined globally for symmetric spaces) such that for each geodesic $c$ with $c(0)=p$ we have $I_{p}(c(t))=c(-t)$ for all $t \in \mathbb{R}$. The symmetric spaces of negative curvature come in 4 families of metrics on $\mathbb{R}^{n}$; the constant curvature metrics (all $n)$, the complex hyperbolic spaces $(n=2 m)$, the quaternionic hyperbolic spaces (for $n=4 m$ ), and the hyperbolic Cayley plane $(\mathrm{n}=16)$. A locally symmetric space is a Riemannian manifold whose universal cover is a symmetric space. They are also characterized as those metrics whose Riemannan curvature tensor is parallel.

The volume entropy $\operatorname{Ent}(g)$ of a Riemannian manifold is the exponential growth rate of the volume, $\operatorname{Vol}(B(p, r))$, of the metric ball of radius $r$ in the universal covering space centered at a lift of $p$. More precisely:

$$
\operatorname{Ent}(g)=\lim _{r \rightarrow \infty}\left(\frac{\log (\operatorname{Vol}(B(p, r)))}{r}\right) .
$$

It is not hard to see that this is independent of the base point $p$. Another equivalent definition (which is not hard to see) is that $\operatorname{Ent}(g)$ is the infimum of $s$ such that

$$
\int_{\tilde{M}} e^{-s d(p, q)} d q<\infty
$$

where $p$ is a fixed point in the universal cover, $\tilde{M}$, and the integral with respect to the Riemannian volume form $d q$.

When $(M, g)$ is a compact manifold of negative curvature (see [Man]) or more generally has no conjugate points (see [Fr-Ma]) then $\operatorname{Ent}(g)$ is just the topological entropy of the geodesic flow.

Let $\left(M, g_{0}\right)$ be a compact manifold with a locally symmetric Riemannian metric $g_{0}$ of negative curvature. Although the theorem of Besson, Courtois, and Gallot in [B-C-G2] is more general, for our purposes we need only consider the case of a different metric $g_{1}$ on $M$. (The general version involves maps from $\left(M_{1}, g_{1}\right)$ to $\left(M_{0}, g_{0}\right)$ of arbitrary degree.)

Theorem 6.1. If $\left(M^{n}, g_{0}\right), n \geq 3$, is a locally symmetric space of negative curvature and $g_{1}$ is another metric on $M$ then

$$
\operatorname{Ent}\left(g_{0}\right) \operatorname{Vol}\left(g_{0}\right)^{\frac{1}{n}} \leq \operatorname{Ent}\left(g_{1}\right) \operatorname{Vol}\left(g_{1}\right)^{\frac{1}{n}} .
$$

Further, if equality holds then $g_{0}$ is isometric to $g_{1}$.

A number of important rigidity conjectures (e.g., the Lichnerowitz conjecture and rigidity of negatively curved manifolds with smooth Anosov splitting) were resolved by this theorem in [B-C-G2]. The consequence of this theorem proved in [B-C-G2] relevant to our paper is that if a compact Riemannian manifold $(M, g)$ has a geodesic flow that is $C^{0}$ conjugate to the geodesic flow of a locally symmetric space $\left(N, g_{0}\right)$ and if the volumes agree then they are isometric. Thus as pointed out in section 4 this implies that if the conjugacy is $C^{1}$ then the volume assumption can be dropped. 
However, even more can be said if both spaces have negative curvature and one is locally symmetric. Hamenstädt [Ha2] has shown that in this case the volumes must be the same and hence the assumption on the volumes can be dropped. Thus one gets:

Theorem 6.2. Compact locally symmetric spaces of negative curvature are $C^{1}$ conjugacy rigid and $C^{0}$ conjugacy rigid among negatively curved spaces.

Cutting and pasting yields (as was presumably known to the authors of [B-C-G2]).

Corollary 6.3. Compact subdomains of symmetric spaces of negative curvature are boundary rigid.

Proof. Let $\left(M, \partial M, g_{0}\right) \subset N$ where $N$ is a symmetric space of negative curvature. Let $\Gamma$ be a cocompact discrete subgroup of the isometry group of $N$ (i.e., $N / \Gamma$ is a compact locally symmetric space) such that for every $\gamma \in \Gamma, \gamma(M) \cap M=\emptyset$. (This can be done since by a result of Malcev [Mal] every finitely generated linear group is residually finite. Thus if $D$ is the diameter of $M$ and we start with any discreet cocompact action by a $\Gamma_{0}$ we can choose a subgroup $\Gamma$ such that for all $x \in N$ and $\gamma \in \Gamma$ $d(x, \gamma(x)) \geq D$.) This implies that a copy of $\left(M, \partial M, g_{0}\right)$ lies in the compact locally symmetric space $N / \Gamma$. The rest of the argument is just the cut and paste construction described in section 4 . The volume is the same since the boundary distance functions are the same by Lemma 2.3. The entropy is the same since the geodesic flows are conjugate. One can also see this directly from the alternative definition of entropy by noting by the triangle inequality that for any $p$ and $q$ in the universal cover $N$ we have $\left|d_{0}(p, q)-d(p, q)\right| \leq 2 D$ for $D$ the diameter of $M$, where $d$ is the distance in the metric induced from the pasted metric on $N / \Gamma$. (Notice that the original metric on $N$ will be changed on countably many copies $M$.) One thing to worry about is that the resulting space will not a-priori be $C^{\infty}$. However the rigidity proof in [B-C-G2] is robust enough to carry through in this case.

The ideas in [B-C-G2] were extended in [Co-Fa] to prove rigidity for some products of rank one symmetric spaces of nonpositive curvature.

In [Gro] Gromov introduced the notion of filling volume, $\operatorname{FillV} \operatorname{Vol}\left(N^{n}, d\right)$, for a compact $^{1}$ manifold $N$ with a metric $d$ (here $d$ is a distance function which is not necessarily Riemannian). To see the actual definition one should see [Gro], but it is shown in [Gro] that when $n \geq 3$

$$
\operatorname{FillVol}\left(N^{n}, d\right)=i n f_{g} \operatorname{Vol}\left(M^{n+1}, g\right),
$$

where $M$ is any manifold such that $\partial M=N$ (one can even take $M=N \times[0, \infty)$ ), the infimum is taken over all Riemannian metrics $g$ on $M$ for which the boundary distance function is $\geq d$.

\footnotetext{
${ }^{1}$ There are also generalizations to the noncompact case (see [Gro]).
} 
Remark 6.4. In the case where $d$ is the boundary distance function of some Riemannian $\left(M^{n+1}, g_{0}\right)$ one can not only fix the topology of $M^{n+1}$ one can also restrict to metrics $g$ which agree with $g_{0}$ when restricted to $N$.

To see this let $g$ be a Riemannian metric on $M$ whose boundary distance function, $d_{g}$ satisfies $d_{g} \geq d$ and consider the metrics on $N \times[0,1]$ defined by

$$
g_{e}=\left.(1-t) g\right|_{N}+\left.t g_{0}\right|_{N}+\epsilon^{2} d t^{2} .
$$

The volume of $g_{\epsilon}$ goes to 0 as $\epsilon$ goes to 0 , while $\left.g_{\epsilon}\right|_{N \times\{0\}}=\left.g\right|_{N}$ and $\left.g_{\epsilon}\right|_{N \times\{1\}}=\left.g_{0}\right|_{N}$. Thus we can define new metrics $\bar{g}_{\epsilon}$ on $M=M \cup N \times[0,1]$ by gluing on a collar with arbitrarily small volume. (The lack of differentiability at the old boundary is not a problem since one can smooth without changing the volumes or distances much.) The only thing to check is that $d_{g_{\epsilon}} \geq d$. To see this first note that $\left.g\right|_{N} \geq\left. g_{0}\right|_{N}$ since $d_{g} \geq d$. Thus on the collar $g_{\epsilon} \geq\left. g_{0}\right|_{N}+\epsilon^{2} d t^{2}$. This means that any curve in the collar from $(p, s)$ to $(q, t)$ has length greater than or equal to $d(p, q)$. This together with the fact that $d_{g} \geq d$ implies that $d_{g_{\epsilon}} \geq d$.

We will call $(M, g)$ a filling of $\left(\partial M, d_{g_{0}}\right)$ if both $d_{g} \geq d_{g_{0}}$ and $\left.g\right|_{\partial M}=\left.g_{0}\right|_{\partial M}$.

Question 6.5. Let $\left(\Omega, g_{0}\right)$ be a compact (or simple) subdomain of a simply connected space of nonpositive (or negative) curvature. Is

$$
\operatorname{FillVol}\left(\partial \Omega, d_{g_{0}}\right)=\operatorname{Vol}\left(\Omega, g_{0}\right) ?
$$

Further if $(\bar{\Omega}, g)$ is a filling with $\operatorname{Vol}(g)=\operatorname{Vol}\left(g_{0}\right)$ must $(\bar{\Omega}, g)$ be isometric to $\left(\Omega, g_{0}\right)$ ?

The rigidity case above would imply the boundary rigidity of $\left(\Omega, g_{0}\right)$. In [Gro] Gromov showed that the answer was yes for subdomains of $\mathbb{R}^{n}$ (in fact for more general flat manifolds) which is how subdomains of $\mathbb{R}^{n}$ were first proved to be boundary rigid.

Another consequence of Theorem 6.1 (which was also pointed out in [Iv]) is:

Corollary 6.6. The answer to the above question is yes for subdomains of symmetric spaces of negative curvature.

Proof. We use the cut and paste argument of the previous corollary replacing the metric $g_{0}$ in a subdomain of a locally symmetric space with $g$. There is again more than one way to compare the entropies of $g$ and $g_{0}$, but we will look at the alternative definition given above as in the proof of the previous lemma. In this case triangle inequalities (and the condition on the boundary distance functions) yield $d(p, q) \geq$ $d_{0}(p, q)-2 D$ for some fixed number $D$. Hence we can conclude that $\operatorname{Ent}(g) \leq \operatorname{Ent}\left(g_{0}\right)$. Hence by Theorem $6.1 \operatorname{Vol}(g) \geq \operatorname{Vol}\left(g_{0}\right)$. The rigidity in Theorem 6.1 yields the rigidity here as in the previous corollary.

Note that the result in section 3 says that if we restrict our "fillings" to metrics that are pointwise conformal to $g_{0}$ (i.e. the only other metrics we consider are of the 
form $\left.g_{1}=f^{2}(x) g_{0}\right)$ then $d_{g_{1}} \geq d_{g_{0}}$ implies $\operatorname{Vol}\left(g_{1}\right) \geq \operatorname{Vol}\left(g_{0}\right)$ with equality if and only if $f \equiv 1$.

The case of surfaces (two dimensions) was taken up in [Iv]. In two dimensions the filling volume question is a little different then in higher dimensions since the topology of the "filling" can affect the minimum of the area. The main result in [Iv] shows that for simple metrics $\left(D, g_{0}\right)$ on the 2-disk $D$ then any metric $g_{1}$ on $D$ such that $d_{g_{1}} \geq d_{g_{0}}$ we have $\operatorname{Vol}\left(g_{1}\right) \geq \operatorname{Vol}\left(g_{0}\right)$.

A result in [C-D-S] gives a local answer to the question for simple metrics of negative curvature. In the statement below $C^{3, \alpha}\left(S^{2} \tau_{M}^{\prime}\right)$ represents the $C^{3, \alpha}$ topology with respect to a fixed underlying metric on the space of symmetric 2-tensors .

Proposition 6.7. For any simple metric $g_{0}$ of negative curvature there is a neighborhood $W \subset C^{3, \alpha}\left(S^{2} \tau_{M}^{\prime}\right)$ of $g_{0}$, with any $0<\alpha<1$, such that if a metric $g_{1} \in W$ induces the same Riemannian metric on the boundary as $g_{0}$ and $d_{g_{1}} \geq d_{g_{0}}$ then $\operatorname{Vol}\left(g_{1}\right) \geq \operatorname{Vol}\left(g_{0}\right)$ with equality if and only if $g_{1}$ is isometric to $g_{0}$.

In fact the curvature condition above can be substantially relaxed (see the chapter by Sharafutdinov in this book).

By analogy with the above, it is natural to pose the following question (which would resolve the local rigidity question and hence yield finiteness theorems as in section 4):

Question 6.8. Let $g_{0}$ be a metric of negative curvature on a compact manifold (without boundary). Is there a $C^{k}$ neighborhood, $W$, of $g_{0}$ such that for all $g_{1} \in$ $W \cap\left\{g_{1} \mid \operatorname{MLspec}\left(g_{1}\right) \geq \operatorname{MLspec}\left(g_{0}\right)\right\}$ then $\operatorname{Vol}\left(g_{1}\right) \geq \operatorname{Vol}\left(g_{0}\right)$ with equality holding if and only if $g_{1}$ is isometric to $g_{0}$ ?

In fact one could ask the question for $g_{1}$ a more general metric of negative curvature. This will be taken up in a future joint work of the author with Dairbekov where the two dimensional inequality should be resolved along with the $n$-dimensional case when $g_{1}=f^{2} g_{0}$.

\section{Nonpositive curvature in higher dimensions}

The condition of nonpositive curvature plays a role in many of the rigidity results we have discussed. In this section we will discuss some results where all the manifolds are assumed to have nonpositive curvature.

The first rigidity result of this type is the "higher rank rigidity theorem". The rank, $\operatorname{Rank}(M)$ of such a space, $M$, is the minimum (over all geodesics) of the dimension of the space of parallel Jacobi fields along a geodesic (it is always $\geq 1$ ). $\operatorname{Rank}(M) \geq 2$ can be expressed in terms of the existence of flat half planes in the universal cover (e.g. see the theorem below). 
The initial proof of the result was due to Ballmann [Ba] and independently by Burns and Spatzier [Bu-Sp]. It was later extended by many others. The version below is proved in [Eb-He] (The result in [Eb-He] is somewhat more general than finite volume.):

Theorem 7.1. Let $M$ be a finite volume nonpositively curved Riemannian manifold and $\tilde{M}=X_{0} \times X_{1} \times X_{2} \times \ldots \times X_{k}$ the de Rham decomposition of the universal cover $\tilde{M}$ (with $X_{0}$ the Euclidean part). Then each $X_{i}(i \geq 1)$ is either an irreducible symmetric space or it contains a geodesic that does not bound a flat half plane in $X_{i}$.

The corresponding result for conjugacy rigidity $[\mathrm{C}-\mathrm{E}-\mathrm{K}]$ is:

Theorem 7.2. If $(M, g)$ and $\left(M_{1}, g_{1}\right)$ are compact manifolds of nonpositive curvature with $\operatorname{rank}(M) \geq 2$, and $\left(M_{1}, g_{1}\right) \in \mathcal{F}^{0} U((M, g))$ then $g_{1}$ is isometric to $g$. Further, when $n \geq 3$ the isometry above can be chosen to induce the "same" action on the fundamental group as the conjugacy $F$.

The easiest nontrivial examples of nonpositively curved manifolds that have rank 1 but whose fundamental group is not hyperbolic (and hence have no metrics of negative curvature) are the so called graph manifolds. Though there have been a number of definitions of "graph manifold" we will use a the rather general one used in [Le]: a graph manifold is a 3 dimensional Haken manifold each of whose components under the minimal decomposition (in the sense of [Ja-Sh, Jo]) is Seifert fibered. The decomposition is by cutting along embedded incompressible tori and Klein bottles. Not all such manifolds admit metrics of nonpositive curvature (see [Le] and [Bu-Ko]) but we will only be concerned with the ones that admit nonpositively curved Riemannian metrics. The following is proved in [Cr1].

Theorem 7.3. If $(M, g)$ and $\left(M_{1}, g_{1}\right)$ are compact nonpositively curved graph manifolds and $\left(M_{1}, g_{1}\right) \in \mathcal{F}^{0} U((M, g))$ then $g_{1}$ is isometric to $g$.

One way to construct some such graph manifolds of nonpositive curvature is to use two building blocks $B_{i}=\Sigma_{i}^{2} \times S^{1}$ where $\Sigma_{i}^{2}$ is a surface of nonpositive curvature with a boundary consisting a closed geodesic (to get smooth results assume that the metric is flat in a neighborhood of the boundary). Notice that the boundary is a totally geodesic flat torus. We can thus glue two such blocks together by reversing the two factors in the boundary tori (assuming the appropriate lengths are compatible) yielding a manifold without boundary of nonpositive curvature. One can easily generalize this to many blocks $B_{i}$ where the surfaces $\Sigma_{i}$ have many geodesics on the boundary.

The topology of graph manifolds forces metrics of nonpositive curvature to have a very restricted geometric form as studied in [Sch] and [Le]. They are the union over totally geodesic boundaries of (one or more) pieces (the "geometric Seifert components") whose universal covers are isometric to a Riemannian product $X \times \mathbb{R}$ where $X$ is a surface of nonpositive curvature with geodesic boundary. They thus become amenable to some techniques developed for the results in section 9 . 


\section{The linearized and local problem}

The deformation boundary rigidity problem attempts to answer the question: Can one deform the metric on a simple (or SGM) manifold $(M, \partial M, g)$ keeping the boundary distance function fixed? In other words: If $g_{t}$ is a 1-parameter family of metrics on $M$ with $g_{0}=g$ such that $d_{g_{t}}=d_{g}$, must there be diffeomorphisms $\phi_{t}$ leaving $\partial M$ pointwise fixed such that $\phi_{t}^{*}\left(g_{t}\right)=g$ ? If this is true then $(M, \partial M, g)$ is called deformation boundary rigid.

Linearizing this problem about $g_{0}$ leads to the following question: If $T$ is a symmetric $n$-tensor such that for every geodesic segment $\gamma$ from boundary point to boundary point we have

$$
0=I_{\gamma}(T) \equiv \int_{0}^{L(\gamma)} T\left(\gamma^{\prime}(t), \gamma^{\prime}(t), \ldots, \gamma^{\prime}(t)\right) d t
$$

then $T=d \omega$ for a an $(n-1)$ symmetric tensor $\omega$. In the above the operator $d$ is the symmetrized covariant derivative (see [Cr-Sh] for more details). The metric case corresponds to the question for symmetric 2-tensors (representing in our case $\frac{d}{d t} g_{t}$ ) and we call a metric $g_{0}$ which satisfies the above for 2-tensors infinitesimally rigid. The map $\gamma \rightarrow I_{\gamma}(T)$ is called the ray transform of $T$. In a separate chapter in this book Sharafutdinov goes into more detail and surveys the known results in this area and in particular discusses how infinitesimal rigidity can yield deformation rigidity via ideas of [Gu-Ka1]. Here we will only mention a few of the results. We will stick to the case of negative curvature and simple metrics (in the "with boundary" case) even though all of the results have been improved to hold for wider classes (e.g. the geodesic flow is Anosov, or subdomains of simple metrics). The reader is referred to the other chapter for these improved results. Our purpose here is to show how one can sometimes use such infinitesimal rigidity results along with symmetry to get global rigidity results. The results of that nature stated here can of course be improved using the improvements covered in the other chapter.

We start with the theorem of Pestov and Sharafutdinov (see [Pe-Sh]);

Theorem 8.1. If $(M, \partial M, g)$ is simple and has negative curvature then it is deformation boundary rigid.

The proof of this theorem uses the Pestov identity which is one of the fundamental tools in the study of the linearized and local versions of these problems.

The analogue of this theorem for manifolds without boundary is [Cr-Sh]:

Theorem 8.2. Compact negatively curved manifolds are spectrally rigid.

It would possibly be better to say "deformation spectrally rigid" but the above terminology was introduced in [Gu-Ka1]. What it says is that if $g_{t}$ is a smoothly varying family of Riemannian metrics on a compact manifold such that $g_{0}$ has negative curvature and for all $t$ the eigenvalue spectrum of $g_{t}$ is the same as that of $g_{0}$, then $g_{t}$ is isometric to $g_{0}$. 
Guillemin and Kazhdan had proved this result in two dimensions [Gu-Ka1] and in the $n$-dimensional case when $g_{0}$ satisfied a pointwise pinched negative curvature condition $[\mathrm{Gu}-\mathrm{Ka} 2]$. Min-Oo [MO] had extended this to the case where $g_{0}$ has negative definite curvature operator.

The proof also yields the corresponding rigidity for the length spectrum (i.e. if the set of lengths of closed geodesics are the same for the $g_{t}$ then they are isometric) and hence the marked length spectrum. Both of the above theorems come from versions that concern symmetric $n$-tensors (not only 2-tensors) as above. Other consequences (extensions of results in [Gu-Ka2]) which are in some sense global rigidity results can be found in [Cr-Sh]:

Theorem 8.3. Let $(M, g)$ be a compact negatively curved Riemannian manifold with simple length spectrum, and $\Delta: C^{\infty}(M) \rightarrow C^{\infty}(M)$ be the corresponding LaplaceBeltrami operator. If real functions $q_{1}, q_{2} \in C^{\infty}(M)$ are such that the operators $\Delta+q_{1}$ and $\Delta+q_{2}$ have coincident spectra, then $q_{1} \equiv q_{2}$.

In the above simple length spectrum means that no two closed geodesics (traversed once) have lengths that are rational multiples of each other. This is a generic condition.

Corollary 8.4. Let $M$ be a compact negatively curved manifold and $f: M \rightarrow \mathbb{R} a$ $C^{\infty}$ function. If $f$ integrates to zero along every closed geodesic then $f$ must itself be zero. In particular a function is determined by its integral along closed geodesics.

For the rest of this section I want to discuss some rigidity consequences of Theorem 8.1 that were developed by the author and Kleiner but never published.

Proposition 8.5. Let $(M, \partial M, g)$ be simple and have negative curvature. If $V$ is a tangent vector field to $M$ defined along $\partial M$ such that for every geodesic segment $\gamma:[0,1] \rightarrow M$ with $\gamma(0)$ and $\gamma(1)$ in $\partial M$, we have

$$
<\gamma^{\prime}(0), V(\gamma(0))>=<\gamma^{\prime}(1), V(\gamma(1))>
$$

then there is an extension of $V$ to a Killing vector field on $M$.

A Killing field, $V$, is an infinitesimal isometry in the sense that the local one parameter group of local diffeomorphisms, $V^{t}$, that it generates are local isometries. Of course any Killing field when restricted to $\partial M$ must satisfy the above condition by the first variation formula. Killing fields are precisely those fields $V$ which satisfy $L_{V} g=0$ or, equivalently, whose dual one form $\omega$ satisfies $d \omega=0$ (with the $d$ from above). If $g_{1}$ is a metric with $d_{g_{1}}=d_{g}$ then for each $g$-Killing field $V$ the proposition yields a $g_{1}$-Killing field $\bar{V}$ with the same (in the sense of section 2 ) restriction to the boundary.

The proposition follows from the version of Theorem 8.1 for one forms: 
Proof. Let $W$ be an arbitrary $C^{\infty}$ extension of $V$ and $\omega$ its dual one form. Our condition implies that $d \omega$ integrates to 0 along every geodesic segment. The analogue of Theorem 8.1 gives us a one form $\bar{\omega}$ which is 0 at $\partial M$ and such that $d \omega=d \bar{\omega}$. Hence $d(\omega-\bar{\omega})=0$ and the dual of $\omega-\bar{\omega}$ is our required Killing field.

Let $(M, \partial M, g)$ be a simple space of negative curvature with a Killing field $V$ and let $\left(M_{1}, \partial M, g_{1}\right)$ also have negative curvature and $d_{g}=d_{g_{1}}$. Then the above gives us a Killing field $V_{1}$ on $M_{1}$ with the same boundary values as $V$. Now if for some open $U \subset \partial M$ the flow of both $V$ and $V_{1}$ are defined for $t \in[0, \epsilon)$ then the neighborhoods $\left\{V^{t}(p) \in M \mid t \in[0, \epsilon)\right.$ and $\left.p \in U\right\}$ and $\left\{V_{1}^{t}(p) \in M \mid t \in[0, \epsilon)\right.$ and $p \in U\}$ are isometric via the map $f$ defined by $f\left(V^{t}(p)\right)=V_{1}^{t}(p)$. This follows since $\left.D f\right|_{V^{t}(p)}$ is the composition of three linear isometries $\left.D V^{-t}\right|_{V^{t}(p)}$, the identification of $T_{p} M$ with $T_{p} M_{1}$, and $\left.D V_{1}^{t}\right|_{p}$. Note here that for any other Killing field $W$ and its corresponding Killing field $W_{1}$ we have $D f(W)=W_{1}$ by the uniqueness properties of Killing fields (if the $C^{1}$ jets of two Killing fields agree at a point then they agree everywhere in a connected set).

It is not hard to extend the argument to see that if each point in $M$ can be written as $V^{t}(p)$ then $g_{1}$ is isometric to $g$. In fact more is true. On a space $(M, \partial M, g)$ we say that $m \in M$ is "reachable from the boundary via Killing fields" if there is a finite sequence $\left\{\left(V_{i}, t_{i}\right) \mid i=1 . . k\right\}$ of Killing fields $V_{i}$ and real numbers $t_{i}$ such that

$$
m=V_{k}^{t_{k}}\left(V_{k-1}^{t_{k-1}}\left(\ldots V_{1}^{t_{1}}(p) \ldots\right)\right)
$$

for some $p \in \partial M$ where $V_{i}^{t}$ represents the local flow generated by $V_{i}$ for time $t$. One can extend the above reasoning to get the following rigidity result:

Corollary 8.6. If $(M, \partial M, g)$ is simple, has negative curvature, and every $m \in M$ is reachable from the boundary via Killing fields then it is boundary rigid among negatively curved spaces.

We will say a space $(M, g)$ (with or without boundary) is a space of revolution if there is a point $o$ (the "origin") such that if $I_{o}$ is the connected subgroup of the isometry group of $M$ that fixes $o$ then the set of differentials acting on $T_{o} M$ is the full group $S O(n)$. Another way of saying this is that in normal polar coordinates $(r, \Theta)$ the metric has the form $d s^{2}=d r^{2}+g^{2}(r) d \Theta^{2}$ where $d \Theta^{2}$ is the standard metric on the $n-1$ sphere and $g: \mathbb{R}^{+} \rightarrow \mathbb{R}^{+}$is 0 only at 0 .

Let $D$ be a convex subdomain of a simply connected negatively curved space of revolution with $o \notin D$. let $q \in \partial D$ be a closest point to $o$, and $L^{n-2}$ be any $(n-2)$ dimensional subspace of the parallel translate, $L^{n-1}$, of $T_{q} \partial D$ along a minimizing geodesic to $o$. Then, since $\exp _{o}\left(L^{n-1}\right) \cap D=\emptyset$, the Killing field that comes from the rotation leaving $L^{n-2}$ fixed will work in the above to show that $D$ is boundary rigid among negatively curved spaces.

Another quite different way to use Proposition 8.5 on spaces of revolution is the following which generalizes to higher dimensions a two dimensional result proved in [Ar-Mi]: 
Corollary 8.7. Let $(M, \partial M, g)$ be a simple manifold of negative curvature which is a space of revolution, then it is boundary rigid among negatively curved spaces. Further, any simple manifold of negative curvature $(M, \partial M, g)$ satisfying

- $\left.g\right|_{\partial M}$ is a round metric on the $n-1$ sphere.

- $d_{g}(p, q)=f(\bar{d}(p, q))$ where $f: \mathbb{R}^{+} \rightarrow \mathbb{R}^{+}$is a function and $\bar{d}$ is the distance function with respect to the boundary sphere metric.

must in fact be a space of revolution.

We note that all spaces of revolution clearly satisfy the two conditions in Corollary 8.7. Since this corollary does not appear in the literature we provide a proof here.

Proof. We do the second part first. Let $(M, \partial M, g)$ have negative curvature and satisfy the two conditions, and let $V$ be a Killing vector field to $\partial M$ coming from the action of $S O(n-1)$ on $\partial M$. We can of course think of $V$ as a tangent vector field to $M$ along $\partial M$. Since for every $p$ and $q$ in $\partial M, \bar{d}\left(V^{t}(p), V^{t}(q)\right)=\bar{d}(p, q)$ we have $d_{g}\left(V^{t}(p), V^{t}(q)\right)=d_{g}(p, q)$ and $\left\langle\gamma^{\prime}(p), V(p)>=<\gamma^{\prime}(q), V(q)>\right.$ for the (unique by simplicity) geodesic segment, $\gamma$ from $p$ to $q$ by the first variation formula. Thus by Proposition 8.5 every such Killing field extends to a Killing field on $M$. Integrating these fields we see that the action of $S O(n-1)$ on the boundary extends to an isometric action on all of $M$. For the rest of the argument we show in the next paragraph that there is an origin $o$.

The normal geodesic leaving any boundary point $p$ must exit at the antipodal point to $p$ and normal to the boundary since the whole geodesic will be fixed by any isometry that leaves $p$ fixed. Let $2 r$ be the (constant by the homogeneity of the boundary) length of these normal geodesic segments. The obvious map $H: \partial M \times[0,2 r] \rightarrow M$ must have some singular points or else it would be a double cover of $M$ which is diffeomorphic to a ball since it is simple. Such a singular point is a focal point $\gamma\left(t_{0}\right)$, of the boundary along some normal geodesic $\gamma$. The focal point has index $n-1$ because of the isometries that fix $\gamma$ and hence is the only focal point along this geodesic by the curvature assumption. In particular $d H_{\left(\gamma(0), t_{0}\right)}(V)=0$ for all vectors tangent to $\partial M \times\left\{t_{0}\right\} \subset \partial M \times[0,2 r]$ at $\left(\gamma(0), t_{0}\right)$. The Jacobi fields along $\gamma$ defining this focal point are the same Jacobi fields along $\gamma$ that define the boundary focal points to $\gamma(2 r)$ along $-\gamma$ since all normal geodesic segments are normal at both endpoints. This means that the distance to the focal locus from $\gamma(2 r)$ along $-\gamma$ is $2 r-t_{0}$. The isometry group forces the same picture along every normal geodesic. In particular $t_{0}=2 r-t_{0}$ and so the focal point occurs at distance $r$ from the boundary along every normal geodesic. Thus the focal locus $H(\partial M \times\{r\})$ is a single point $o$, our origin, since $d H$ is 0 on all tangent vectors to $\partial M \times\{r\}$.

Now to see the first part let $\left(M_{1}, \partial M, g_{1}\right)$ have negative curvature and $d_{g_{1}}=d_{g}$. Then it satisfies the two conditions above so by the part already proven $\left(M_{1}, \partial M, g_{1}\right)$ is a space of revolution. That means, in particular, that for every 2-plane $P$ in $T_{o} M_{1}, \exp _{o}(P)$ is a totally geodesic submanifold and hence has negative curvature. The corresponding surface in $M$ (i.e. the totally geodesic surface having the same boundary circle) will also have negative curvature and the same boundary distance 
function. Hence the surfaces are isometric by section 5. But that means that $g(r)=$ $g_{1}(r)$ (where $g(r)$ comes from the metric representation $d s^{2}=d r^{2}+g^{2}(r) d \Theta^{2}$ ) and thus the spaces are isometric.

\section{Products}

The only cases of rigidity in higher dimensions without any curvature assumptions involve manifolds that have some sort of product structure. In [Cr-Kl1] it was shown that all compact Riemannian manifolds $X$ are stably $C^{1}$-conjugacy rigid in the sense that $X \times S^{1}$ is $C^{1}$ conjugacy rigid. More generally

Theorem 9.1. Let $M$ be a compact Riemannian manifold with a nontrivial parallel vector field. Then $M$ is $C^{1}$ conjugacy rigid.

In $X \times S^{1}$ the parallel vector field points in the $S^{1}$ direction. In general, by the DeRham splitting theorem, if $M$ admits a parallel vector field then the universal cover of $M$ splits off a line (though $M$ itself need not be a product). Surprisingly it is still not known if $M$ is $C^{1}$ conjugacy rigid if rather than a parallel vector field $M$ admits a parallel line field (hence a double cover admits a parallel vector field). Of course the double covers will be isometric but there is no guarantee that the isometry will commute with the covering maps.

The idea behind the proof of Theorem 9.1 is to study Jacobi fields (or more precisely Jacobi tensors) perpendicular to the "vertical" direction. Using an extremal property of Jacobi fields and the fact that the volume is the same it is shown that the conjugacy must map the parallel vector field in one space to a parallel vector field in the other. This uses the $C^{1}$ conjugacy. That reduces the problem to the case where the universal covers of both manifolds are Riemannian products.

Now if two manifolds are nontrivial products [Cr-Kl1] studies $C^{0}$ conjugacies between the geodesic flows. The techniques developed here were later applied in [C-E-K] and [Cr1] (also see section 7). In general one needs to assume an additional assumption that the conjugacy "preserves angles". For a Riemannian product $M_{1} \times \ldots \times M_{k}$, we define $\alpha=\left(\alpha_{1}, \ldots, \alpha_{k}\right): U\left(M_{1} \times \ldots \times M_{k}\right) \longrightarrow R^{k}$ by $\alpha_{i}(v)=\left|\pi_{i *}(v)\right|$ where $\pi_{i}: M_{1} \times \ldots \times M_{k} \longrightarrow M_{i}$ is projection onto the $i$ th factor. The most general result is:

Proposition 9.2. Let $M=M_{1} \times M_{2}$ and $N=N_{1} \times N_{2}$ be Riemannian products such that $\operatorname{dim}\left(M_{i}\right) \neq 0$. If there is a uniform conjugacy $F: U M \rightarrow U N$ which preserves $\alpha$ then $M$ is isometric to $N$.

In the above $M$ and $N$ are not assumed to be compact, but the conjugacy is assumed to be uniformly continuous. In many cases it is not hard to prove that a conjugacy preserves $\alpha$. A particular consequence is: 
Proposition 9.3. Let $M=M_{1} \times M_{2}$ be a compact Riemannian product such that $\operatorname{dim}\left(M_{i}\right) \neq 0$, and let $N=X_{1} \times X_{2} \times \ldots \times X_{k}$ where each $X_{i}$ is a compact Riemannian manifold of negative curvature (or more generally a manifold with Anosov geodesic flow). If there is a $C^{0}$ conjugacy $F: U M \rightarrow U N$ then $M$ is isometric to $N$.

\section{No conjugate points}

One definition of "no conjugate points" for a Riemannian manifold $(M, g)$ without boundary is that in the universal cover all geodesics are minimizing. The official definition is that no Jacobi field along any geodesic vanishes twice. The easiest examples of manifolds without conjugate points are the nonpositively curved ones. There are other examples (see for example [Gul]), however, there is no example known of a compact manifold that admits a metric with no conjugate points metric that does not also admit a different metric with nonpositive curvature.

The purpose of this section is to survey the rigidity results related to the no conjugate points condition. It is a natural condition for our setting in that (see [Cr-Kl1]) it is preserved by $C^{0}$ conjugacies, i..e, if $(M, g)$ and $\left(M_{1}, g_{1}\right)$ have $C^{0}$ conjugate geodesic flows and $(M, g)$ has no conjugate points then $\left(M_{1}, g_{1}\right)$ has no conjugate points. On the other hand rigidity results are hard to come by since the condition is not a convexity condition as curvature conditions are, but only a global length minimization condition.

The most celebrated such rigidity result is the so called E. Hopf conjecture solved by Burago and Ivanov [Bu-Iv1] (The two dimensional case had been solved by Hopf $[\mathrm{Ho}])$ :

Theorem 10.1. A metric on the $n$-torus without conjugate points is flat.

One could use this to conclude that any SGM subdomain of a compact flat manifold is boundary rigid. Another way of seeing this had been found in [Cr2]. For $x \in M$ let $\operatorname{Vol}(B(\bar{x}, r))$ be the volume of the ball of radius $r$ in the universal cover of $M$ centered at a lift $\bar{x}$ of $x$. This is well defined since all such balls have the same volume. It was shown in [Cr2] that if one fixes the radius $r>0$ then the average over $x$ (with respect to the standard volume on $M$ ) of $\operatorname{Vol}(B(\bar{x}, r)$ ) is greater than or equal to the volume of the flat ball of radius $r$ with equality if and only if the metric is flat. Also in [Cr2] is a more general result relating the metric entropy to the average volume of balls. To get the boundary rigidity for SGM submanifolds [Cr2] considers the asymptotics as $r$ goes to $\infty$.

If $\left(M, g_{0}\right)$ is a complete Riemannian manifold satisfying some property $\mathcal{P}$ (such as "no conjugate points"), then we will say that $\left(M, g_{0}\right)$ is rigid under compactly supported perturbations (respecting the property $\mathcal{P}$ ) if any metric $g_{1}$ on $M$ which satisfies property $\mathcal{P}$ and coincides with $g_{0}$ outside a compact set $K \subset M$ is isometric to $g_{0}$. 
Euclidean space was shown to be rigid under compactly supported perturbations with nonpositive (or nonnegative) sectional curvature in [Gr-Wu]. In fact they are rigid under compactly supported perturbations having no conjugate points (see [Gr-Gu] for the two dimensional case and [Cr2] in general).

In $[\mathrm{Cr}-\mathrm{K} 12]$ this was extended to:

Theorem 10.2. Let $X$ be a 1-connected Riemannian manifold without conjugate points, and let $g_{0}$ be the Riemannian product metric on $X \times \mathbb{R}$. Then $g_{0}$ is rigid under compactly supported perturbations having no conjugate points.

In the proof, one first shows that if the metric is changed in a compact region without introducing conjugate points then the boundary distance function of the region does not change. One then proves boundary rigidity for such regions to get the result.

The case where $X$ above is not simply connected was considered in [Cr-Kl3]. This case poses many difficulties not seen in the simply connected case. In particular, so far one cannot handle spaces whose fundamental groups grow too fast. Never-the-less many cases are proven in [Cr-Kl3]. One result is:

Theorem 10.3. Let $\left(M, g_{0}\right)$ be a complete flat manifold. Then $g_{0}$ is rigid under compactly supported perturbations having no conjugate points.

The case where $M$ is compact relies on Theorem 10.1 while the non compact case comes from [Cr-Kl3].

\section{Some Other Related Topics}

There are many related topics that we have not taken up here. We mention a few briefly now.

One might ask why one should consider Riemannian metrics rather than say Finsler metrics (i.e. there is a Banach norm in each tangent space rather than an inner product). The boundary rigidity problem in general is under-determined so one cannot expect uniqueness. Arcostanzo in [Ar] shows among other things how one can explicitly construct many Finsler metrics on the 2-disk with the same boundary distance function as the flat ball. Thus one needs to add extra conditions to have any chance. One natural condition to consider is when the curvature is parallel. Two places where such rigidity results are proved are $[\mathrm{Ki}-\mathrm{Yi}]$ and $[\mathrm{Fo}]$. Another example where rigidity (in the sense of Theorems 5.1 and 5.2) fails to hold if the metric is not assumed to be Riemannian can be found in [Bo]. Some nice results of filling volume type (as in section 6) for Finsler metrics can be found in [Iv] and [Bu-Iv2].

Without curvature assumptions there are now many examples of isospectral metrics and isospectral deformations. See for example the chapter in this book by Ballmann. Nilpotent groups and their quotients have served as a rich source of examples of nontrivial isospectral deformations. However there is conjugacy rigidity inside this 
class (both manifolds assumed to be nilmanifolds) see [G-M-S, Eb, Fa]. A nice survey of this subject can be found in [Go-Go].

Rigidity questions for Lorentz metrics has been studied in [A-D-H]. While [Grog] considers magnetic flows rather than geodesic flows.

\section{References}

[A-D-H] L. Andersson, M. Dahl, and R. Howard, Boundary and lens rigidity of Lorentzian surfaces, Trans. Amer. Math. Soc. 348 (1996) no. 6, 2307-2329.

[An] M. Anderson, Remarks on the compactness of isospectral sets in low dimensions, Duke Math. J. 63 (1991), 699-711.

[Ar] M. Arcostanzo, Des métriques finslériennes sur le disque à partir d'une fonction distance entre les points du bord, Comm. Math. Helv. 69 (1994), 229-248.

[Ar-Mi] M. Arcostanzo, and R. Michel, Métriques de révolution d'un disque et invariance par rotation de la longueur des géodésiques, Geom. Dedicata 76 (1999) no. 2, 197-209.

[Ba] W. Ballmann, Nonpositively curved manifolds of higher rank, Annals of Math (2) 122 (1985), 597-609.

[Be] M. Berger, Une bourne inférieure pour le volume d'une variété riemannienne en function du rayon d'injectivité, Ann. Inst. Fourier (Grenoble) 30 (1980) no.3, 259-265.

[Be-Ka] M. Berger and J. L. Kazdan, A Sturm-Liouville inequality with applications to an isoperimetric inequality for volume in terms of injectivity radius, and to Wiedersehen manifolds, in General Inequalities 2 (Proceedings of the second International Conference on General Inequalities, 1978), E. F. Beckenbach (ed.), ISNMA47, Birkhauser Verlag, no.3, 251-254.

[B-C-G1] G. Besson, G. Courtois, and S. Gallot, Entropies et rigidités des espaces localement symétriques de courbure strictement négative, Geom. Funct. Anal. 5 (1995) no. 5, 731-799.

[B-C-G2] G. Besson, G. Courtois, and S. Gallot, Minimal entropy and Mostow's rigidity theorems, Ergodic Theory Dynam. Systems 16 (1996) no. 4, 623-649.

[Bo] F. Bonahon, Surfaces with the same marked length spectrum, Topology Appl. 50 (1993) no. 1, 55-62.

[B-P-P] R. Brooks, P. Perry, and P. Petersen, Compactness and finiteness theorems for isospectral manifolds, J. Reine Angew. Math. 426 (1992), 67-89.

[Br] R. Brooks, Cheng's theorem in $L^{p}$ spectral geometry, Algebra i Analiz 8 (1996) no. 2, 98-102; translation in St. Petersburg Math. J. 8 (1997), no. 2, 255-258. 
[Bu-Iv1] D. Burago and S. Ivanov, Riemannian tori without conjugate points are flat, Geom. Funct. Anal. 4 (1994) No. 3, 259-269.

[Bu-Iv2] D. Burago and S. Ivanov, On asymptotic volume of Finsler tori, minimal surfaces in normed spaces, and symplectic filling volume, (preprint).

[Bu-Ka] K. Burns and A. Katok, Manifolds with non-positive curvature, Ergod. Th. \& Dynam. Syst. 5 (1985), 307-317.

[Bu-Sp] K. Burns and R. Spatzier, Manifolds of nonpositive curvature and their buildings, Inst. Hautes Études Sci. Publ. Math. 65 (1987), 35-59.

[Bu-Ko] S.V. Buyalo and V. L. Kobelskii, Geometrization of graph-manifolds. II. Isometric geometrization (Russian) Algebra i Analiz 7, no. 3 (1995), 96-117; translation in St. Petersburg Math. J. 7 (1996) no. 3, 387-404.

[Cao] J. Cao, Rigidity for non-compact surfaces of finite area and certain Kähler manifolds, Ergodic Theory Dynam. Systems 15 (1995) no.3, 475-516.

[Ch-Ya] S.-Y. Chang and P. Yang, Isospectral conformal metrics on 3-manifolds, J. Amer. Math. Soc. 3 (1990) no.3, 117-145.

[Co-Fa] C. Connell and B. Farb, Minimal entropy rigidity for lattices in products of rank one symmetric spaces, (preprint).

[Cr1] C. Croke, Conjugacy rigidity for nonpositively curved graph manifolds, (preprint).

[Cr2] C. Croke, Rigidity and the distance between boundary points, J. Diff. Geom. 33 (1991), 445-464.

[Cr3] C. Croke, Rigidity for surfaces of non-positive curvature, Comm. Math. Helv. 65 (1990) no.1, 150-169.

[Cr4] C. Croke, Volumes of balls in manifolds without conjugate points, International J. Math. vol.3 ( 1992) no.4, 455-467.

[C-D-S] C. Croke, N. Dairbekov, and V. Sharafutdinov, Local boundary rigidity of a compact Riemannian manifold with curvature bounded above, Trans. Amer. Math. Soc. 325 (2000) no.9, 3937-3956.

[C-E-K] C. Croke, P. Eberlein, and B. Kleiner, Conjugacy and rigidity for nonpositively curved manifolds of higher rank, Topology 35 (1996), 273-286.

[C-F-F] C. Croke, A. Fathi, and J. Feldman, The marked length-spectrum of a surface of nonpositive curvature, Topology 31 (1992) no. 4, 847-855.

[Cr-Kl1] C. Croke and B. Kleiner, Conjugacy and Rigidity for Manifolds with a Parallel Vector Field, J. Diff. Geom. 39 (1994), 659-680.

[Cr-K12] C. Croke and B. Kleiner, A rigidity theorem for simply connected manifolds without conjugate points, Ergod. Th. \& Dynam. Syst. 18 (1998) pt. 4, 807812. 
[Cr-Kl3] C. Croke and B. Kleiner, A rigidity theorem for manifolds without conjugate points, Ergod. Th. \& Dynam. Syst. 18 (1998) pt. 4, 813-829.

[Cr-Sh] C. Croke and V. Sharafutdinov, Spectral rigidity of a compact negatively curved manifold, Topology 37 (1998), 1265-1273.

[Du-Gu] J. Duistermaat and V. Guillemin, The spectrum of positive elliptic operators and periodic bicharacteristics, Invent. Math. 29 (1975) no. 1, 39-79.

[Eb] P. Eberlein, Geometry of 2-step nilpotent groups with a left invariant metric, Ann. Sci. École Norm. Sup. (4) 27 (1994) no. 5, 611-660.

[Eb-He] P. Eberlein and J. Heber, A differential geometric characterizationof symmetric spaces of higher rank, Inst. Hautes Études Sci. Publ. Math. 71 (1990), $33-44$.

[Fa] H.-R. Fanai, Conjugaison géodśique des nilvariétés de rang deux (French), J. Lie Theory 10 (2000) no. 1, 147-155.

[Fe-Or] J. Feldman and D. Ornstein, Semi-rigidity of horocycle flows over surfaces of variable negative curvature, Ergod. Th. \& Dynam. Syst. 7 (1987), 49-74.

[Fo] P. Foulon, locally symmetric Finsler spaces in negative curvature, (preprint).

[Fr-Ma] A. Freire and R. Mañé, On the entropy of the geodesic flow in manifolds without conjugate points, Invent. Math. 69 (1982), 375-392.

[Go-Go] C. Gordon and R. Gornet, Spectral geometry on nilmanifolds, Progress in inverse spectral geometry, 23-49, Trends Math., Birkhäuser, Basel, 1997.

[G-M-S] C. Gordon, Y. Mao, and D. Schueth, Symplectic rigidity of geodesic flows on two-step nilmanifolds, Ann. Sci. École Norm. Sup. (4) 30 (1997) no. 4, 417-427.

[Go-Wi] C. Gordon and E. Wilson, Isospectral deformations of compact solvmanifolds, J. Diff. Geom. 19 (1984), 241-256.

[Gr-Gu] L. Green and R. Gulliver, Planes without conjugate points, J. Diff. Geom. 22 (1985), 43-47.

[Gr-Wu] R. Greene and H. H. Wu, On a new gap phenomenon in Riemannian Geometry, Proc. Nat. Acad. Sci. U.S.A. 79 (1982) no. 2, part 1, 714-715.

[Grog] S. Grognet, Flots magnétiques en courbure négative (French), Ergodic Theory Dynam. Systems 19 (1999) no. 2, 413-436.

[Gro] M. Gromov, Filling Riemannian manifolds, J. Diff. Geom. 18 (1983), 1-147.

[Gu-Ka1] V. Guillemin and D. Kazhdan, Some inverse spectral results for negatively curved 2-manifolds, Topology 19 (1980), 301-312. 
[Gu-Ka2] V. Guillemin and D. Kazhdan, Some inverse spectral results for negatively curved n-manifolds, Proc. Sympos. Pure Math. 36, Amer. Math. Soc., Providence RI, 1980, 153-180.

[Gul] R. Gulliver, On the variety of manifolds without conjugate points, Trans. Amer. Math. Soc. 210 (1975), 185-201.

[Ha1] U. Hamenstädt, Time-preserving conjugacies of geodesic flows, Ergod. Th. \& Dynam. Sys. 12 (1992), 67-74.

[Ha2] U. Hamenstädt, Cocycles, symplectic structures and intersection, Geom. Funct. Anal. 9 (1999) no. 1, 90-140.

[Her] G. Herglotz, Uber die Elastizitaet der Erde bei Beruecksichtigung ihrer variablen Dichte, Zeitschr. fur Math. Phys. 52 (1905), 275-299.

[Ho] E. Hopf, Closed surfaces without conjugate points, Proc. Nat. Ac. Sc. USA 34 (1948), 47-51.

[Iv] S. Ivanov, On two-dimensional minimal fillings, (preprint).

[Ja-Sh] W. Jaco and P. Shalen, Seifert fibred spaces in 3-manifolds, Mem. Amer. Math. Soc. 220 (1979).

[Jo] K. Johansson, Homotopy equivalences of 3-manifolds with boundary, Springer LNM 761 (1979).

[Ki-Yi] C-W Kim and J-W Yim, Rigidity of noncompact Finsler manifolds, Geom. Dedicata 81 (2000) no. 2, 245-259.

[L-S-U] M. Lassas, V. Sharafutdinov, and G. Uhlmann, Semiglobal rigidity for Riemannian metrics, (preprint).

[Le] B. Leeb, 3-manifolds with(out) metrics of nonpositive curvature, Invent. Math. 122 (1995), 277-289.

[Man] A. Manning, Topological entropy for geodesic flows, Ann. of Math. (2) 110 (1979) no. 3, 567-573.

[Mal] A. Mal'cev, On isomorphic matrix representations of infinite groups (Russian) Mat. Rec.Math[Mat.Sbornik]N.S. 8(50) (1940), 405-422.

[Mu1] R. G. Mukhometov, Inverse kinematic problem of seismic on the plane, Math. Problems of geophisics, Akad. Nauk. SSSR, Sibirsk.Otdel., Vychisl. Tsentr, Novosibirsk, 6 (1975) no.2, 243-252 (Russian).

[Mu2] R. G. Mukhometov, The reconstruction problem of a two-dimensioina; Riemannian metric, and integral geometry, Soviet Math. Dokl. 18 (1977) no. 1, $27-37$.

[Mu3] R. G. Mukhometov, On a problem of reconstructing Riemannian metrics, Siberian Math. J. 22 (1982) no. 3, 420-433. 
[Mi] R. Michel, Sur la rigidité imposée par la longuer des géodésiques, Inv. Math. 65 (1981), 71-83.

[MO] M. Min-Oo, Spectral rigidity for manifolds with negative curvature operator, Contemp. Math. 51 (1986), 99-103.

[Ot1] J.-P. Otal, Le spectre marqué des longueurs des surfaces à courbure négative, Ann. of Math. 131 (1990), 151-162.

[Ot2] J.-P. Otal, Sur les longueurs des géodésiques d'une métrique à courbure négative dans le disque, Comment. Math. Helv, 65 (1990) no. 2, 334-347.

[Pe-Sh] L. Pestov and A. Sharafutdinov, Integral geometry of tensor fields on a manifold of negative curvature, Novosibirsk (transl. from Sibirskii Math. Zhurnal 29 (1988) No. 3, 114-130).

[Ro1] V.G. Romanov, Reconstructing a function by means of integrals along a family of curves, Sibirsk. Mat. Zh. 8 (1967) no. 5, 1206-1208.

[Ro2] V.G. Romanov, Integral geometry on geodesics of an isotropic Riemannian metric, Soviet Math. Dokl. 19 (1979) no. 4, 847-851.

[Sch] V. Schroeder, Rigidity of nonpositively curved graphmanifolds, Math. Ann. 274 (1986), 19-26.

[Vi] M. Vignéras, Variétés riemanniennes isospectrales et non isométriques, Ann. of Math. 110 (1980), 21-32.

[We] A. Weinstein, Fourier integral operators, quantization and the spectra of Riemannian manifolds, Colloques Internationaux C.N.R.S., no. 273- Géométrie symplectique et physique mathematique (1975), 289-298.

[Wi-Zo] E. Wiechert and K. Zoepprtz, Uber Erdbebenwellen, Nachr. Königl. Geselschaft Wiss, Göttingen 4 (1907), 415-549. 